\title{
Does CRP predict outcome in bipolar disorder in regular outpatient care?
}

\author{
Sonya M. Balukova, Bartholomeus C. M. Haarman*, Rixt F. Riemersma-van der Lek and Robert A. Schoevers
}

\begin{abstract}
Background: The association between inflammation and the course of mood disorders is receiving increased attention. This study aims to investigate whether a sub-group of patients with BD can be identified for which a higher CRP (C-reactive protein) level at baseline is associated with an unfavorable prognosis.

Methods: This is a historic cohort study using CRP at baseline, with 15-month follow-up of mood status and medication. Cross-sectional analyses include boxplots, one-way ANOVA, receiver operating characteristics (ROC) curve and Chi square test, and the longitudinal analysis using multivariate Cox-regression.

Results: Eighty-four bipolar disorder patients were included in the analyses. Cross-sectionally, no statistically significant difference was found in CRP distribution across mood states $(p=0.372)$ or rapid cycling state $(p=0.656)$. Also, no CRP cut-off level was distinguished between euthymic and non-euthymic patients according to the ROC curve ( $p=0.449, \mathrm{AUC}=0.452,95 \% \mathrm{Cl} 0.327,0.576)$, and a literature-derived cut-off value ( $3 \mathrm{mg} / \mathrm{L}$ ) again demonstrated no difference $(p=0.530)$. Longitudinally, no association was found between CRP and prognosis of disease neither in euthymic $[-2 \log$ likelihood $=120.460 ; C R P: p=0.866, B=-0.011, \mathrm{OR}=0.989$ (95\% Cl 0.874-1.120)] nor noneuthymic patients [( -2 log likelihood $=275.028 ; C R P: p=0.802, B=0.010, \mathrm{OR}=1.010$ (95\% Cl 0.937-1.088)]. Medication use did not affect these associations.

Conclusions: We found no statistically significant association between CRP and a more unfavorable BD prognosis, suggesting that the application of CRP as a practical biomarker to predict outcome in a naturalistic outpatient care setting is not as straightforward as it may seem.
\end{abstract}

Keywords: C-reactive protein, Bipolar disorder, Inflammation, Biological markers, Prognosis, Historic cohort

\section{Background}

Bipolar disorder (BD) is associated with a significant decrease in quality of life and social functioning of patients (ten Have et al. 2002). Despite the availability of pharmacological treatment, its efficacy is far from optimal (Burcusa and Iacono 2007; Frecska et al. 2012). A promising approach for optimizing the treatment is to tailor it to the specific characteristics of a patient as part of a 'personalized medicine' approach which encompasses not only psychological but also biological markers (Hamdani et al. 2013).

\footnotetext{
*Correspondence: b.c.m.haarman@umcg.nl

Department of Psychiatry, CC44, University of Groningen, University

Medical Centre Groningen, P.O. Box 30.001, 9700 RB Groningen,

The Netherlands
}

There is increasing evidence to suggest that immunological processes may contribute to the emergence as well as the prognosis and severity of BD (Liu et al. 2004). Apart from pro-inflammatory cytokines such as IL-2 and IL-6 (Brietzke et al. 2009), C-reactive protein (CRP) is an acute phase protein that is produced in response to infection and inflammation. It is also considered to be another candidate biomarker for detecting immune dysregulation in BD. A number of studies suggest an association between CRP and mood disorders, especially during a manic episode (Maes et al. 1995; Dickerson et al. 2007; Cunha et al. 2008).

Based on the idea that immunological processes play a role in the pathophysiology of $\mathrm{BD}$, it can be hypothesized that an increased activity of these processes, measured with CRP, would lead to more instability in BD 
symptomatology and course of disease. Increased inflammatory activity has been shown to be related to therapy resistance and chronicity in unipolar depression (Sluzewska et al. 1997; Miller et al. 2009; Raison et al. 2013). Recently, Becking et al. (2013) demonstrated an increased CRP to predict future development of manic symptoms in a sample of MDD (major depressive disorder) patients, also suggesting that this is a subtype with an untoward prognosis. However, to date, no studies have examined this issue in bipolar patients.

The current study investigated whether, in a clinical setting, higher CRP levels at baseline may predict a worse BD outcome, defined as a shorter time to relapse (if euthymic) or a longer time to recover. As some medication may influence inflammatory processes, this was taken into account.

\section{Methods}

\section{Participants and ethical considerations}

For the present historic cohort study, we used medical files from 84 BD patients from the BD outpatient department of the Psychiatry Department of the University Medical Center Groningen (UMCG), The Netherlands.

Patients, who provided a written informed consent to participate, were included if the following criteria were fulfilled: DSM-IV-TR diagnosis of BD, age between 18 and 65 , recorded CRP value, not pregnant or less than 6 months postpartum, no current serious somatic illnesses (current infections or liver disease, serious un- or undertreated heart, lung or neurological disorders).

All patient data were collected as part of regular outpatient care and were anonymously used for research according to the Data Protection Act (WBP) and Medical Treatment Agreement (WGBO), as formulated in the Code of Conduct for the Use of Data in Health Research, also known as the Research Code of Conduct (see also IRB in Additional file 1).

\section{Assessments of study parameters}

The study parameters included CRP, measurement date and value, BD type, presence of a rapid cycling course, and mood episodes and medication 1 month before and 15 months following the CRP measurement.

Assessment of the psychiatric condition of patients was determined by the first author based on information from two sources: the electronic medical records of the treating psychiatrists and the lifechart methodology (LCM) records (a systematic collection of data on the course of illness and treatment presented in a graphical form) (Denicoff et al. 2000). The condition was noted as one of five categories: (0) Euthymia; (1) Depressive episode; (2) Hypomania or mania; (3) Mixed episode; (4) Unstable mood. Long-lasting clinically significant mood instability that did not fulfill the criteria for any mood episode was assessed as an "unstable" mood, while subthreshold symptoms were assessed as no change in episode (Perlis et al. 2006).

Serum CRP (high sensitive CRP) is routinely measured at hospital admission in this Psychiatry Department (and additional measurements are performed when there is a psychiatric or somatic event for a patient). CRP was assayed using a wide range turbidimetric CRP assay (CRPL3 assay) on a Roche Modular platform (Roche, Mannheim, Germany). Starting from the CRP measurement date at baseline ( $T 0)$, the time to episode change (T1) and the corresponding psychiatric condition were gathered from the medical file.

Cases with unclear information and/or diagnosis of current mental status were discussed with the psychiatrists of the BD outpatient department. Remaining decisions were taken in discussion with all authors of this study.

\section{Statistical analysis}

Both cross-sectional and longitudinal analyses were performed. Cross-sectionally, data were visualized using boxplots and tested using histograms, P-P plots, Kolmogorov-Smirnov test, Kruskal-Wallis, a receiver operating characteristics (ROC) curve and Fisher's exact test.

The longitudinal analysis comprised a multivariate Cox-regression based on the time passed until a change of episode has occurred where the main covariate was the scale of CRP values. This analysis was done separately for euthymic patients and those who were in a mood episode at baseline (depressed, manic, mixed, unstable). This was done because the variable signifying elapsed time has a different meaning for patients in a euthymic state than for a non-euthymic one, and should thus be interpreted differently to describe the progression of disease. Longer time before episode change signifies a better BD prognosis if a subject is euthymic (means longer time in remission), but it means a worse course if a subject is non-euthymic (means longer time in an episode).

Medication was added to the Cox-regression to examine whether it affected CRP or changed the effect of $\mathrm{CRP}$ on BD progress. Separate analyses were done after excluding those non-psychopharmaceutical drugs that are known to have anti-inflammatory and/or CRP-affecting properties (O'Brien et al. 2006; Goldstein et al. 2009; Ximenes et al. 2013).

As part of sensitivity analyses, all tests were repeated in each of the following subgroups for each timepoint and then for the whole study: excluding subjects taking antiinflammatory medication; excluding all medication that affects CRP; excluding outliers, defined as CRP values above $10 \mathrm{mg} / \mathrm{L}$. 


\section{Results}

The sample of this study consisted of 84 patients and Table 1 shows their characteristics. Testing the data for normality showed a non-normal distribution of the CRP data, with a positive skewness and a significant difference from a normal distribution seen by Kolmogorov-Smirnov test $[\mathrm{D}(84)=0.258, p<0.001]$. Because of this, median values are provided for each mood state in Table 1.

\section{Cross-sectional analysis}

The differences between the distribution of CRP values among the mood states were first examined using Kruskal-Wallis. No statistical significance was found ( $p=0.372$ ) (see Table 1). Figure 1 shows the distribution of mood episodes at baseline. Using KolmogorovSmirnov, no statistical significant difference was found between the patients that were rapid cycling and patients that were not ( $p=0.656$, see Fig. 2). Repeating the crosssectional analyses excluding all medications that are known to affect CRP, as well as excluding CRP outliers, generally yielded the same, non-significant results.

After excluding six CRP outliers, there were 37 (44\%) who were in euthymia and 47 (56\%) were non-euthymic: $27(32.1 \%)$ were in a depressive episode, $12(14.3 \%)$ in a manic episode, $2(2.4 \%)$ in a mixed episode and $6(7.1 \%)$ were unstable.

Using an ROC curve, the data were tested to identify a cut-off value of CRP which could suggest whether subjects at baseline would be euthymic or in an episode. Based on the curve (Additional file 1: Figure S1), no such cut-off value was found (AUC $=0.452, p=0.449,95 \%$ CI $0.327,0.576$ ).
Consequently, the cross-sectional analyses were performed using a literature-based CRP cut-off value of $3.0 \mathrm{mg} / \mathrm{L}$ and a Chi square test for the not-normal distribution of CRP value. The results showed that, crosssectionally, higher CRPs were almost equally distributed among euthymic and non-euthymic patients (42.1\% euthymic and $57.9 \%$ non-euthymic by CRP $>3 \mathrm{mg} / \mathrm{L}$; $44.6 \%$ euthymic and $55.4 \%$ non-euthymic by $\mathrm{CRP} \leq 3 \mathrm{mg} / \mathrm{L})$. Fisher's exact test confirmed that these results have no statistical significance $(p=0.530)$.

\section{Longitudinal analysis For euthymic subjects at baseline}

From the 37 subjects who were euthymic at baseline, 20 had a change of episode during the trial period, while 17 patients stayed euthymic. The results are shown in Table 2 and the Hazard function of the covariate CRP is in Fig. 3. There is no correlation between CRP value and the event of episode change (relapsing). The odds ratio $(=0.989)$ approaches equality for both groups (results are very close to the neutral line). Moreover, these findings are not statistically significant and so the null hypothesis could not be rejected.

\section{For non-euthymic subjects at baseline}

There were 47 subjects who were in an episode at baseline of which 30 became euthymic, while 17 patients remained in a mood episode during the study period. The results are shown in Table 2 and in Fig. 4. The odds ratio is approaching equality (results are very close to the neutral line) and these findings are not statistically significant. As seen in the euthymic group, these results also do not show an association between CRP and relapsing.

Table 1 Characteristics of patient population

\begin{tabular}{|c|c|c|c|c|c|c|}
\hline Parameter & Euthymic & Depressed & (Hypo)manic & Mixed & Unstable & All \\
\hline Mean age (SD) & $44.9(12.4)$ & $42.7(12.4)$ & $43.2(10.3)$ & $46(7.1)$ & $40.5(10.2)$ & $43.6(11.7)$ \\
\hline Number of subjects (\%) & $37(44.0)$ & $27(32.1)$ & $12(14.3)$ & $2(2.4)$ & $6(7.1)$ & $84(100)$ \\
\hline Male gender (\%) & $11(37.9)$ & $10(34.5)$ & $4(13.8)$ & $2(6.9)$ & $2(6.9)$ & $29(34.5)$ \\
\hline Female gender (\%) & $26(47.3)$ & $17(30.9)$ & $8(14.5)$ & 0 & $4(7.3)$ & $55(65.5)$ \\
\hline Number of subjects with BD type I (\%) & $26(41.9)$ & $21(33.9)$ & $11(17.7)$ & $2(3.2)$ & $2(3.2)$ & $62(100)$ \\
\hline Number of subjects with BD type II (\%) & $7(41.2)$ & $5(29.4)$ & $1(5.9)$ & 0 & $4(23.5)$ & $17(100)$ \\
\hline Number of subjects with rapid cycling (\%) & $4(30.8)$ & $3(23.1)$ & $2(15.4)$ & 0 & $4(30.8)$ & $13(100)$ \\
\hline Number of subjects with metabolic syndrome $(\%)^{b}$ & $9(13.8)$ & $6(9.2)$ & $2(3.1)$ & $1(1.5)$ & $2(3.1)$ & $20(30.8)^{c}$ \\
\hline Median C-reactive protein (mg/L) & 1.10 & 2.10 & 0.90 & 3.08 & 1.39 & 1.37 \\
\hline
\end{tabular}

$S D$ standard deviation, $B D$ bipolar disorder

a Bipolar disorder type information missing for five patients

b Metabolic syndrome information missing for 19 patients (criteria: fasting glucose $\geq 5.6 \mathrm{mmol} / \mathrm{L}$ and two or more of following: $\mathrm{BMI} \geq 30 \mathrm{~kg} / \mathrm{m}^{2}$, hypertriglyceridemia $\geq 1.7 \mathrm{mmol} / \mathrm{L}, \mathrm{HDL}-\mathrm{C}<0.9 \mathrm{mmol} / \mathrm{L}$ in men and $<1.0 \mathrm{mmol} / \mathrm{L}$ in women, hypertension $\geq 140 / 90 \mathrm{mmHg}$ )

c The number in brackets in this cell represents the percentage of all subjects who have metabolic syndrome, while the rest of the percentages in this row represent the distribution of subjects with metabolic syndrome across the mood states 


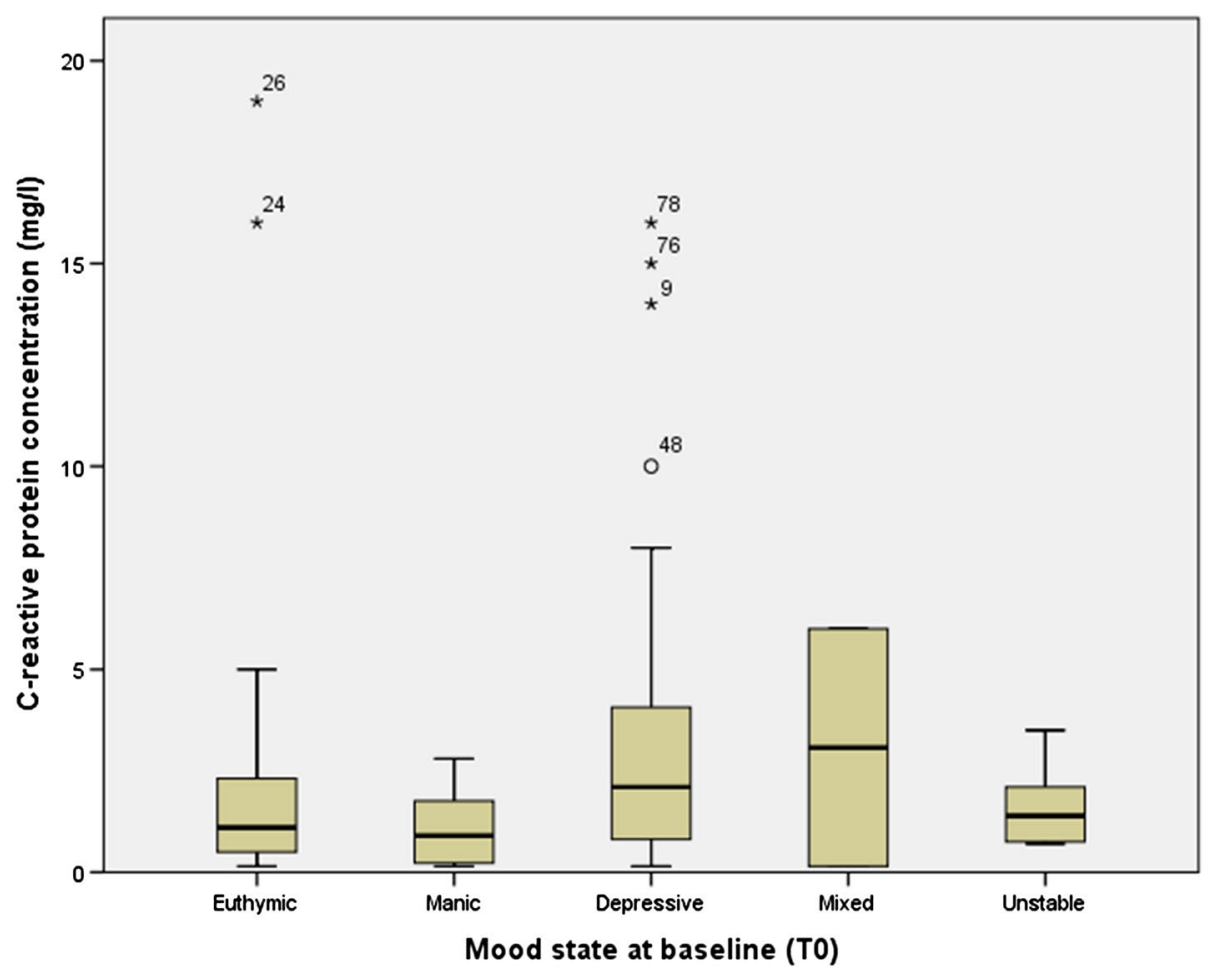

Fig. 1 Boxplot of CRP distribution at baseline across mood state. The $Y$-axis depicts CRP concentration in $\mathrm{mg} / \mathrm{L}$ starting from $0 \mathrm{mg} / \mathrm{L}$ and incrementing with $5 \mathrm{mg} / \mathrm{L}$. In the $X$-axis, bars signify the interquartile range of CRP values in each mood group, and the thickened line inside them shows the median value of CRP within this mood group. The stars and circle show the boxplot outliers, while the numbers next to them signify the specific number of the patient with this outlier value. The circle is a patient with CRP value closest to the threshold value but above it, so it is also not included

After adjusting for covariates and testing for interactions of CRP with medication at the two timepoints, there were no results with statistical significance in each of the two mood-state groups-euthymic and noneuthymic. Adjusting for separate medication types used in any moment during the study again yielded no associations with statistical significance.

Table 3 shows a summary of the used medication at the $T 0$ and $T 1$ timepoints as well as in total for the whole study period.

\section{Discussion}

\section{Principal findings}

To our knowledge, this is the first study examining longitudinal associations between CRP level and clinical outcome in BD patients in a naturalistic treated and reallife measurement outpatient setting. In a first cross-sectional analysis, we could not distinguish a sub-group of BD patients with an elevated baseline CRP level based on affective state or rapid cycling state. In the longitudinal analysis, no statistically significant association was found between higher CRP values and relapsing in either euthymic or non-euthymic patients, as well as when comparing them.

\section{Comparison to previous studies}

The results of the cross-sectional analysis can be compared to several previous studies on the association of CRP with BD. Two of the studies with a total of 202 patients reported that for their BD subjects higher CRP was significantly associated with manic state compared to the other mood episodes (Dickerson et al. 2007; Cunha et al. 2008).

Three other studies with a total of 248 patients, however, did not demonstrate significant differences in CRP across the mood states, which is corresponding to the current study results (Hornig et al. 1998; Hope et al. 2011; Tsai et al. 2012).

The findings of the influence of medication on the reported associations between CRP values and mood states in the above studies are also conflicting. Hornig et al. (1998) found a significant negative interaction between lithium and CRP within BD patients, while Dickerson et al. (2007) found that no medication affected 


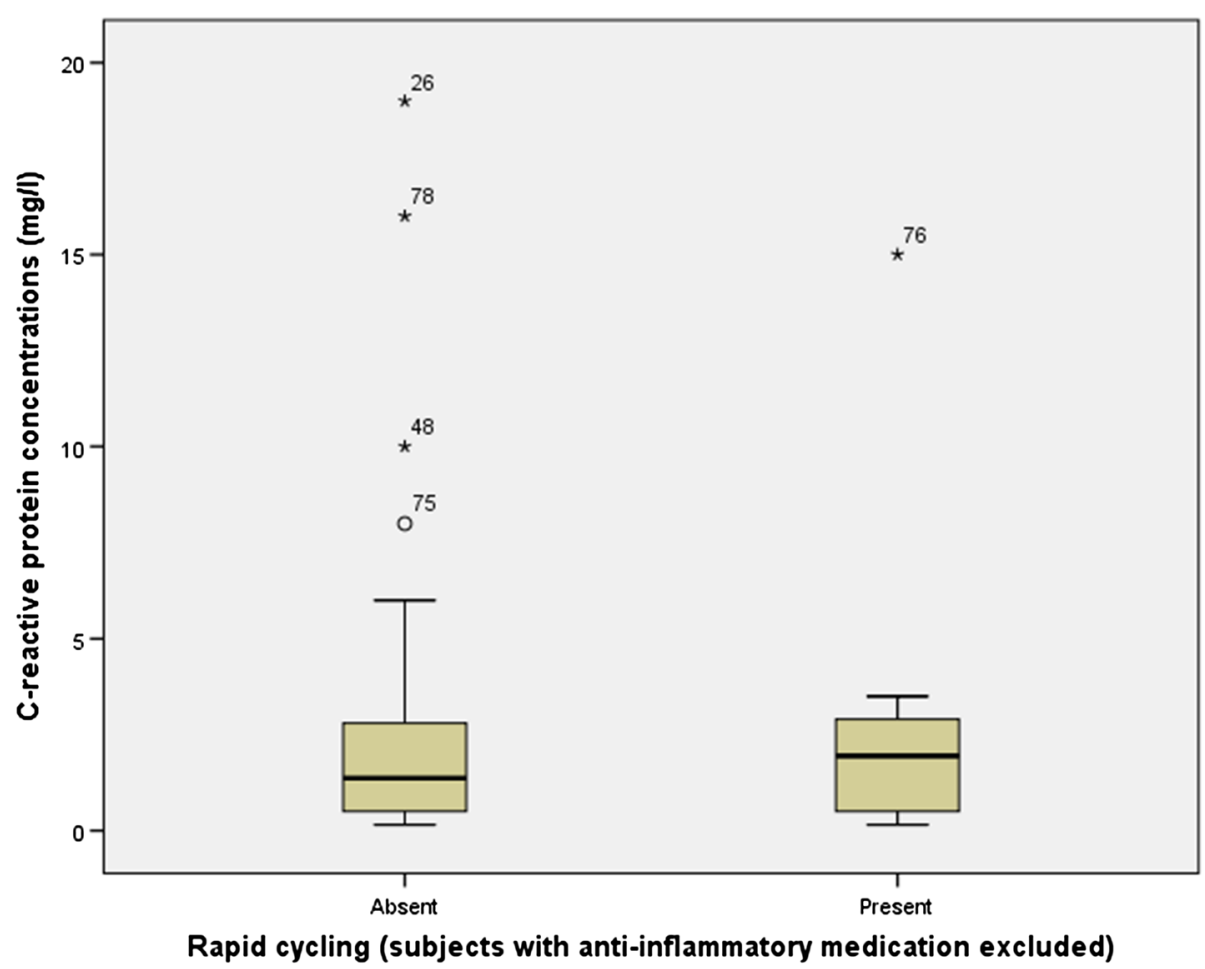

Fig. 2 Boxplot of CRP distribution between patients with and without rapid cycling. The $Y$-axis depicts CRP concentration in $\mathrm{mg} / \mathrm{L}$ starting from $0 \mathrm{mg} / \mathrm{L}$ and incrementing with $5 \mathrm{mg} / \mathrm{L}$. In the $X$-axis, bars signify the interquartile range of CRP values in each patient group, and the thickened line inside them shows the median value of CRP within this mood group. The stars and circle show the boxplot outliers, while the numbers next to them signify the specific number of the patient with this outlier value

Table 2 Longitudinal results from Cox-regression

\begin{tabular}{|c|c|c|c|c|c|c|}
\hline \multirow[t]{3}{*}{ Patient group } & \multicolumn{6}{|c|}{ CRP after adjusting for } \\
\hline & \multirow[t]{2}{*}{-2 log likelihood } & \multirow[t]{2}{*}{ Sig. } & \multirow[t]{2}{*}{ B coef. } & \multirow[t]{2}{*}{ Odds ratio } & \multicolumn{2}{|c|}{$95 \% \mathrm{Cl}$ of odds ratio } \\
\hline & & & & & Lower & Upper \\
\hline Euthymic patients at baseline & 120.460 & 0.866 & -0.011 & 0.989 & 0.874 & 1.120 \\
\hline Non-euthymic patients at baseline & 275.028 & 0.802 & 0.010 & 1.010 & 0.937 & 1.088 \\
\hline
\end{tabular}

the CRP association with $\mathrm{BD}$, as we found in the current study.

In a recent meta-analysis Dargél et al. (2015) demonstrated an overall cross-sectional association between BD and CRP. In this meta-analysis, CRP levels were elevated in manic and euthymic patients compared to $\mathrm{HC}$, but not in depressed BD patients compared to HC. Recent prospective longitudinal studies demonstrated CRP alterations across mood states (Jacoby et al. 2016), as well as before and after various treatments (Uyanik et al. 2015). In addition, an increased CRP was found to be associated with an increased risk for developing late-onset BD in a large Danish demographic sample (Wium-Andersen et al. 2016). Adding to this body of knowledge, the present study shows that using CRP as a practical biomarker to predict outcome in a naturalistic outpatient care setting is not as easily applicable as it may seem.

\section{Limitations}

The present study has several limitations. A limitation pertaining the use of historical data is that CRP measurements were more or less routinely measured, and more in those patients who were psychiatrically or somatically ill. Since it was not purely measured as a routine, a selection bias cannot be ruled out.

Furthermore, body fat percentage, smoking, blood pressure, physical activity and SNP polymorphisms are suggested to be associated with different baseline levels 


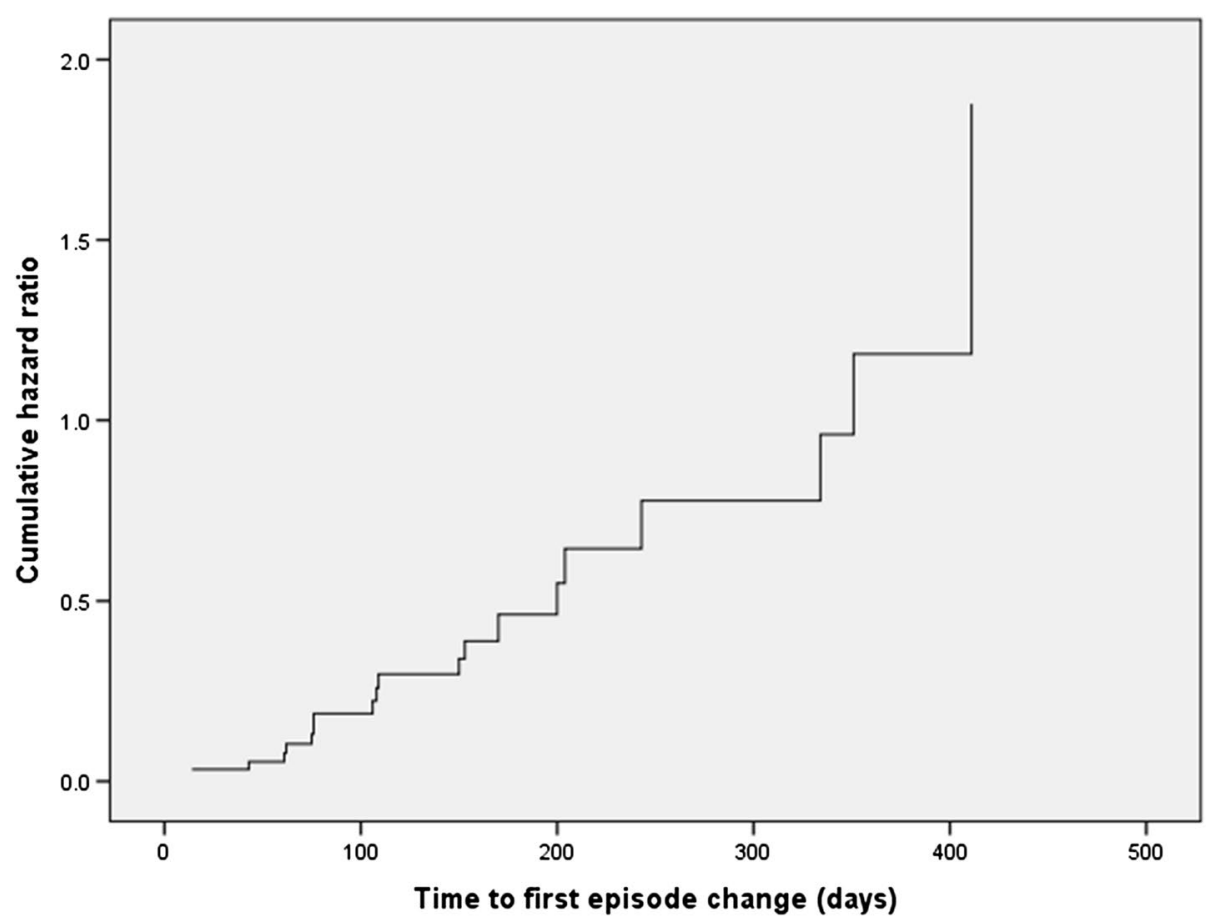

Fig. 3 Hazard function at mean of the covariate CRP for subjects euthymic at baseline. This figure illustrates what the hazard ratio is for relapsing of a subject with a given CRP value compared to a subject with a CRP value of 1 unit lower in the course of the studied period. The $Y$-axis represents the rate of relapsing to a non-euthymic state of all subjects euthymic at baseline. Elapsed time period in days until a change in the mood state has occurred (to either manic, depressed, mixed state or unstable) is depicted on the $X$-axis

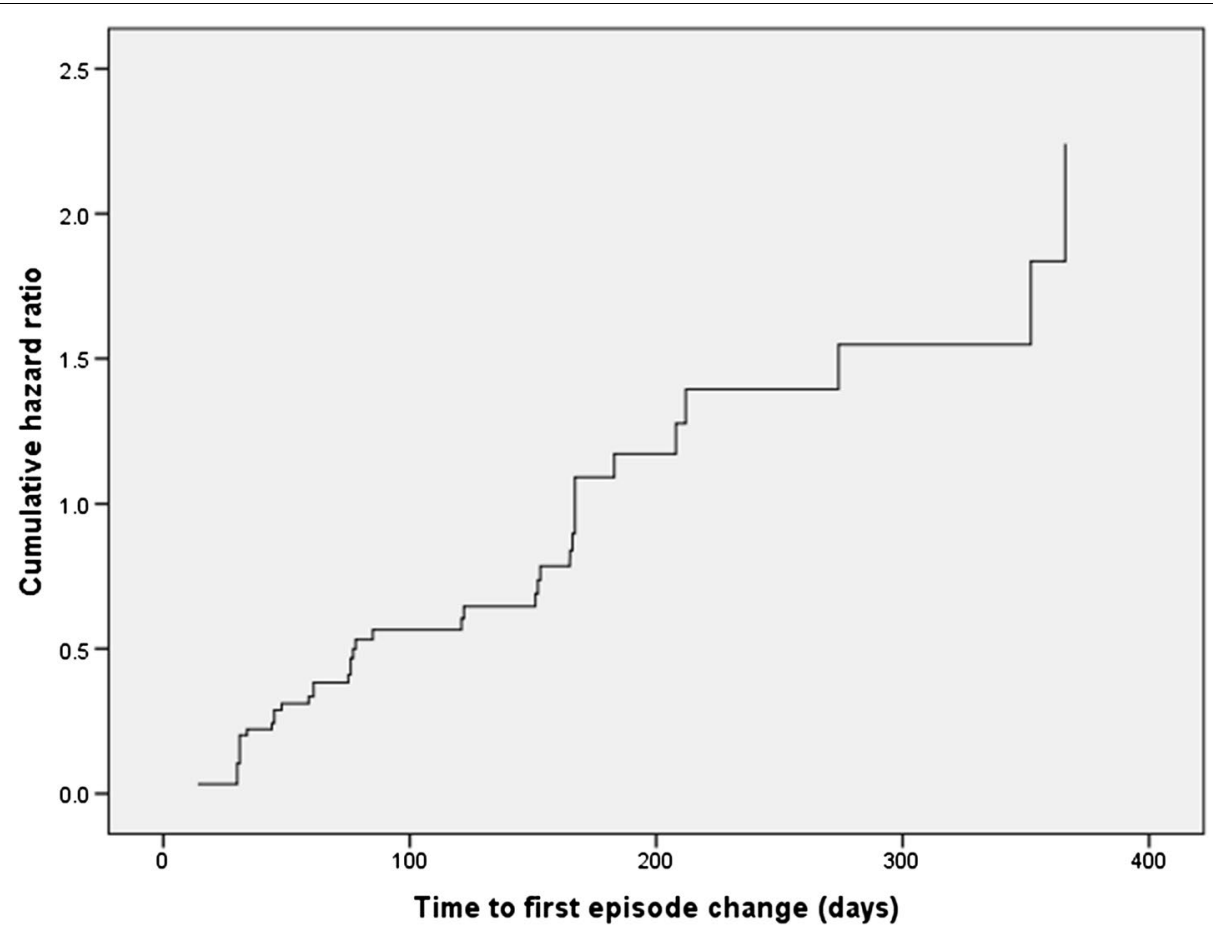

Fig. 4 Hazard function at mean of the covariate CRP for subjects sick at baseline. This figure illustrates what the hazard ratio is for recovering of a subject with a given CRP value compared to a subject with a CRP value of 1 unit lower in the course of the studied period. The Y-axis represents the rate of recovering of all subjects sick at baseline. The elapsed time period in days until an euthymic state has occurred is depicted in the $X$-axis 
Table 3 Number of subjects per medication type

\begin{tabular}{lccc}
\hline Parameter & $\boldsymbol{T O}^{\mathbf{a}}$ & $\boldsymbol{T 1}^{\mathbf{b}}$ & Total $^{\mathbf{c}}$ \\
\hline Lithium (\%) & $31(36.9)$ & $47(56)$ & $48(57.1)$ \\
Valproic acid (\%) $^{\text {Anti-inflammatory drugs (\%) }}{ }^{\mathrm{d}}$ & $14(16.7)$ & $9(10.7)$ & $16(19.0)$ \\
Tricyclic antidepressants (TCA) (\%) $^{\mathrm{d}}$ & $12(14.3)$ & $12(14.3)$ & $16(19.0)$ \\
Antidepressants (non-TCA) (\%) & $31(36.9)$ & $27(32.1)$ & $32(38.1)$ \\
Antipsychotics (\%) $_{\text {Benzodiazepines (\%) }}^{35(41.7)}$ & $34(40.5)$ & $39(46.4)$ \\
\hline
\end{tabular}

a Medication used up to and including the baseline (TO)

b Medication used up to and including the time of episode change is such occurred (T1)

c Medication used for the total period of the study ( 1 month before and up to

15 months after CRP measurement, unless episode change occurred earlier)

d Values are the same for both $T 0$ and $T 1$ timepoints

of CRP (Palosuo et al. 1986; Visser et al. 1999; Ford 2002; Davey Smith et al. 2005; Marnell et al. 2005) and in the present study, we were not able to correct for these variations.

Finally, the study would have benefitted from a larger sample size.

\section{Proposed mechanisms}

There are two factors to consider which could play a role in shaping the results from this study: use of medication and the pathophysiological model of BD.

Due to the naturalistic design of this study, a great variety of medications was used, some of which have been suggested to have anti-inflammatory properties. Lithium, part of the standard treatment of dipolar disorder in the Netherlands, was used by more than half of the patients in this study (57.1\%). Although its mechanism of action in bipolar depression is not fully understood yet (Goldstein et al. 2009), several studies have found evidence that lithium causes a decrease in the inflammatory marker CRP (Sluzewska et al. 1997; Hornig et al. 1998) and a similar effect is also described for SSRIs (selective serotonin reuptake inhibitors) used by $38 \%$ of the subjects here (18). However, there are reports that lithium and antidepressants may also exhibit pro-inflammatory properties (Goldstein et al. 2009; Hamer et al. 2011; Nassar and Azab 2014). These properties were postulated after observing the stimulating effect of lithium on pro-inflammatory cytokines such as Tumor Necrosis Factor- $\alpha$ (TNF- $\alpha$ ), Interleukin-4 (IL-4) and Interleukin-6 (IL-6). It is not yet clear what causes these reverse effects. There could be various mechanisms via which medication is influencing CRP. However, at the same time no such effect was demonstrated in this study. Therefore, other factors should be considered.
Our findings therefore suggest that the inflammatory model of $\mathrm{BD}$ is probably much more complex than what can be shown by straightforward inflammatory alterations demonstrated by elevated markers such as CRP (Altamura et al. 2014) at one point in time.

Compared to the inflammatory mechanism in somatic disorders where CRP, being an acute phase protein, directly correlates to acute worsening of a disease, it may be that immunological processes in $\mathrm{BD}$ are affected by more factors and additional mechanisms, which need to be added to the model to gain a better understanding. One such hypothesis is that inflammatory changes in psychiatric disorders are mediated through a shift toward the tryptophan/kynurenine pathway, which leads to the formation of further factors that may affect the functioning of the brain, such as neurotoxic accumulation of the metabolite 3-hydroxykynurenine (Myint and Kim 2013). Another hypothesis is the co-occurrence of autoimmunity alongside immune dysregulation in BD. It is thus likely that BD is related to a state of immune dysregulation, rather than more pure immune activation (Rege and Hodgkinson 2013; Haarman et al. 2014).

It has been suggested that the immune-mediating pathogenesis of BD occurs at a much younger age, and what follows is a dysregulation of the bodily systems. The long-lasting results are then what we see at an older age: unhealthy and unexpected interactions and responses of the whole body, and of inflammatory markers as well (Beumer et al. 2012). Another interesting possibility is that what we see is not necessarily an inflamed but a damaged organism, given that CRP reacts not only to inflammatory stimuli, but also to damaged cells (Kushner et al. 2006).

To increase our understanding of the position of immune-system-mediated pathophysiological processes in $\mathrm{BD}$, it is necessary to measure CRP and other relevant immune bio-assays prospectively in a larger sample size. In that regard, it is important to investigate what happens on an intra-individual level: additional trials are necessary measuring CRP on different timepoints in a prospective longitudinal manner so that individual changes could be followed and analyzed. Perhaps, these studies will elucidate models, adjusting for variables known to influence CRP, that eventually enable CRP to be used as a practical biomarker to predict outcome in naturalistic treatment settings.

\section{Additional file}

Additional file 1. Supplemental information containing additional IRB approval information and Figure S1: ROC curve of CRP tested for euthymic vs. non-euthymic subjects. 


\section{Abbreviations}

BD: bipolar disorder; CRP: C-reactive protein; LCM: lifechart methodology; MDD: major depressive disorder.

\section{Authors' contributions}

All authors contributed to the concept and design of the study. SB, BH contributed in the analyses and interpretation of the data and in the drafting of the manuscript. All authors provided critical revision. All authors read and approved the final manuscript.

\section{Acknowledgements}

We thank Dr. J. E. Kootstra-Ros for her valuable assistance in retrieving archived data.

\section{Competing interests}

The authors declare that they have no competing interests.

\section{Ethics approval and consent to participate}

Please see the "Participants and ethical considerations" section above.

\section{Funding}

No specific funding was provided for this study.

Received: 30 April 2016 Accepted: 25 June 2016

Published online: 18 July 2016

\section{References}

Altamura AC, Buoli M, Pozzoli S. Role of immunological factors in the pathophysiology and diagnosis of bipolar disorder: comparison with schizophrenia. Psychiatry Clin Neurosci. 2014;68(1):21-36.

Becking K, Boschloo L, Vogelzangs N, Haarman BCM, Riemersma-van der Lek R, Penninx BWJH, et al. The association between immune activation and manic symptoms in patients with a depressive disorder. Transl Psychiatry. 2013:3(10):e314.

Beumer W, Gibney SM, Drexhage RC, Pont-Lezica L, Doorduin J, Klein HC, et al. The immune theory of psychiatric diseases: a key role for activated microglia and circulating monocytes. J Leukoc Biol. 2012;92(September):1-17.

Brietzke E, Stertz L, Fernandes BS, Kauer-Sant'Anna M, Mascarenhas M, Escosteguy Vargas A, et al. Comparison of cytokine levels in depressed, manic and euthymic patients with bipolar disorder. J Affect Disord. 2009;116(3):214-7.

Burcusa SL, lacono WG. Risk for recurrence in depression. Clin Psychol Rev. 2007:27:959-85

Cunha AB, Andreazza AC, Gomes FA, Frey BN, da Silveira LE, Gonçalves CA, et al. Investigation of serum high-sensitive $C$-reactive protein levels across all mood states in bipolar disorder. Eur Arch Psychiatry Clin Neurosci. 2008;258(5):300-4.

Dargél AA, Godin O, Kapczinski F, Kupfer DJ, Leboyer M. C-reactive protein alterations in bipolar disorder. J Clin Psychiatry. 2015;76(2):142-50.

Davey Smith G, Lawlor DA, Harbord R, Timpson N, Rumley A, Lowe GD, et al. Association of $C$-reactive protein with blood pressure and hypertension: life course confounding and mendelian randomization tests of causality. Arterioscler Thromb Vasc Biol. 2005;25(5):1051-6.

Denicoff KD, Leverich GS, Nolen WA, Rush AJ, McElroy SL, Keck PE, et al. Validation of the prospective NIMH-Life-Chart Method (NIMH-LCM-p) for longitudinal assessment of bipolar illness. Psychol Med. 2000;30(6):1391-7.

Dickerson F, Stallings C, Origoni A, Boronow J, Yolken R. Elevated serum levels of $C$-reactive protein are associated with mania symptoms in outpatients with bipolar disorder. Prog Neuropsychopharmacol Biol Psychiatry. 2007:31(4):952-5.

Ford ES. Does exercise reduce inflammation? Physical activity and C-reactive protein among US adults. Epidemiology. 2002;13(5):561-8.

Frecska E, Balla P, Falussy L, Ferencz A, Varga Z. The message of the survival curves: I. Composite analysis of long-term treatment studies in bipolar disorder. Neuropsychopharmacol Hungarica. 2012;14:155-64.
Goldstein BI, Kemp DE, Soczynska JK, McIntyre RS. Inflammation and the phenomenology, pathophysiology, comorbidity, and treatment of bipolar disorder: a systematic review of the literature. J Clin Psychiatry. 2009;70(8):1078-90.

Haarman BCM, Riemersma-Van der Lek RF, Burger H, Netkova M, Drexhage RC, Bootsman F, et al. Relationship between clinical features and inflammation-related monocyte gene expression in bipolar disorder-towards a better understanding of psychoimmunological interactions. Bipolar Disord. 2014;16(2):137-50.

Hamdani N, Doukhan R, Kurtlucan O, Tamouza R, Leboyer M. Immunity, inflammation, and bipolar disorder: diagnostic and therapeutic implications. Curr Psychiatry Rep. 2013;15(9):387.

Hamer M, Batty GD, Marmot MG, Singh-Manoux A, Kivimäki M. Anti-depressant medication use and C-reactive protein: results from two populationbased studies. Brain Behav Immun. 2011;25(1):168-73.

Hope S, Dieset I, Agartz I, Steen NE, Ueland T, Melle I, et al. Affective symptoms are associated with markers of inflammation and immune activation in bipolar disorders but not in schizophrenia. J Psychiatr Res. 2011;45(12):1608-16.

Hornig M, Goodman D, Kamoun M, Amsterdam J. Positive and negative acute phase proteins in affective subtypes. J Affect Disord. 1998;49(1):9-18.

Jacoby AS, Munkholm K, Vinberg M, Pedersen BK, Kessing LV. Cytokines, brainderived neurotrophic factor and C-reactive protein in bipolar I disorderresults from a prospective study. J Affect Disord. 2016;197:167-74.

Kushner I, Rzewnicki D, Samols D. What does minor elevation of C-reactive protein signify? Am J Med. 2006;119(2):17-28.

Liu HC, Yang YY, Chou YM, Chen KP, Shen WW, Leu SJ. Immunologic variables in acute mania of bipolar disorder. J Neuroimmunol. 2004;150(1-2):116-22.

Maes M, Smith R, Scharpe S. The monocyte-T-lymphocyte hypothesis of major depression. Psychoneuroendocrinology. 1995;20(2):111-6.

Marnell L, Mold C, Du Clos TW. C-reactive protein: ligands, receptors and role in inflammation. Clin Immunol. 2005:117(2):104-11.

Miller AH, Maletic V, Raison CL. Inflammation and its discontents: the role of cytokines in the pathophysiology of major depression. Biol Psychiatry. 2009;65(9):732-41.

Myint AM, Kim YKKY. Network beyond IDO in psychiatric disorders: revisiting neurodegeneration hypothesis. Neuropsychopharmacology Biol Psychiatry. 2013;48:304-13.

Nassar A, Azab A. Effects of lithium on inflammation. ACS Chem Neurosci. 2014;5:451-8.

O'Brien SM, Scott LV, Dinan TG. Antidepressant therapy and C-reactive protein levels. Br J Psychiatry. 2006;188:449-52.

Palosuo T, Husman T, Koistinen J, Aho K. C-reactive protein in population samples. Acta Med Scand. 1986;220(2):175-9.

Perlis RH, Ostacher MJ, Marangell LB, Hongwei Z, Wisniewski SR, Ketter TA, et al. Predictors of recurrence in bipolar disorder: primary program for bipolar disorder (STEP-BD). Am J Psychiatry. 2006;163(4):217-24.

Raison CL, Rutherford RE, Woolwine BJ, Shuo C, Schettler P, Drake DF, et al. A randomized controlled trial of the tumor necrosis factor antagonist infliximab for treatment-resistant depression: the role of baseline inflammatory biomarkers. JAMA Psychiatry. 2013;70(1):31-41.

Rege S, Hodgkinson SJ. Immune dysregulation and autoimmunity in bipolar disorder: synthesis of the evidence and its clinical application. Aust N Z J Psychiatry. 2013;47(12):1136-51.

Sluzewska A, Sobieska M, Rybakowski JK. Changes in acute-phase proteins during lithium potentiation of antidepressants in refractory depression. Neuropsychobiology. 1997;35(3):123-7.

ten Have M, Vollebergh W, Bijl R, Nolen WA. Bipolar disorder in the general population in The Netherlands (prevalence, consequences and care utilisation): results from The Netherlands Mental Health Survey and Incidence Study (NEMESIS). J Affect Disord. 2002;68(2-3):203-13.

Tsai S-Y, Chung K-H, Wu J-Y, Kuo C-J, Lee H-C, Huang S-H. Inflammatory markers and their relationships with leptin and insulin from acute mania to full remission in bipolar disorder. J Affect Disord. 2012;136(1-2):110-6.

Uyanik V, Tuglu C, Gorgulu Y, Kunduracilar H, Uyanik MS. Assessment of cytokine levels and hs-CRP in bipolar I disorder before and after treatment. Psychiatry Res. 2015:228(3):386-92. 
Visser M, Bouter LM, McQuillan GM, Wener MH, Harris TB. Elevated C-reactive protein levels in overweight and obese adults. JAMA. 1999;282(22):2131-5.

Wium-Andersen MK, Ørsted DD, Nordestgaard BG. Elevated C-reactive protein and late-onset bipolar disorder in 78,809 individuals from the general population. Br J Psychiatry. 2016;208(2):138-45.
Ximenes JCM, de Oliveira Gonçalves D, Siqueira RMP, Neves KRT, Santos Cerqueira G, Correia AO, et al. Valproic acid: an anticonvulsant drug with potent antinociceptive and anti-inflammatory properties. Naunyn Schmiedebergs Arch Pharmacol. 2013;386(7):575-87.

\section{Submit your manuscript to a SpringerOpen ${ }^{\circ}$ journal and benefit from:}

- Convenient online submission

- Rigorous peer review

- Immediate publication on acceptance

- Open access: articles freely available online

- High visibility within the field

- Retaining the copyright to your article 